\title{
Structural and Magnetic Stabilities of Fe Bilayers on $\mathrm{Cu}(111)^{*}$
}

\author{
Katsuya Masuda, ${ }^{\dagger}$ Kohji Nakamura, Toru Akiyama, and Tomonori Ito \\ Department of Physics Engineering, Mie University, \\ 1577 Kurima-machiya, Tsu, Mie 514-8507, Japan \\ (Received 14 December 2013; Accepted 4 February 2014; Published 8 March 2014)
}

\begin{abstract}
Structural and magnetic stability of Fe bilayer films on $\mathrm{Cu}(111)$ substrate was investigated from first-principles calculations, in which two atomic-stackings are considered for the fct-like and bct-like phases of the Fe bilayers. When a ferromagnetic (FM) alignment of the Fe bilayer is assumed, the surface Fe atoms energetically favor the bridge sites (bct-like phase) better than the hollow sites (fct-like phase). On the contrary, for an antiferromagnetic (AFM) alignment, the fct-like phase becomes favorable. The results suggest that the bi-stability of the FM and AFM structures may lead to the coexistence of the fct-like and bct-like phases observed in experiments.

[DOI: 10.1380/ejssnt.2014.102]
\end{abstract}

Keywords: Iron; Magnetic films; Density functional calculations

\section{INTRODUCTION}

Fe thin films have been attracted attention due to their fundamental properties concerning the phase transitions and the rich magnetic behaviors [1]. In epitaxial growth for the Fe thin films, $\mathrm{Cu}$ substrate has been used because the lattice mismatch is small and the magnetism is not influenced by the substrate $[2,3]$. Indeed, the Fe films on the $\mathrm{Cu}(111)$ substrate exhibit a wide variety of atomic and magnetic structures $[4,5]$. Particularly, the Fe bilayer islands are known to show that two different phases coexist [6]; fct-like phase where the surface Fe atoms locate on the hollow sites of the subsurface Fe layer, and bct-like phase where the surface Fe atoms are on the bridge sites. In addition, the positions of the surface Fe atoms have been controlled by applying a high external electric field by using a tip of scanning-tunneling microscope [7, 8], which opens a new way towards electric-field-controlled (a) $\delta=0$

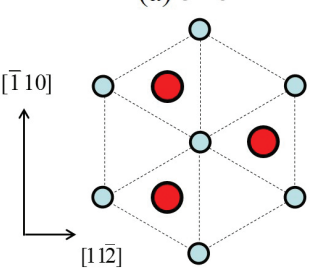

(b) $\delta=0.5$

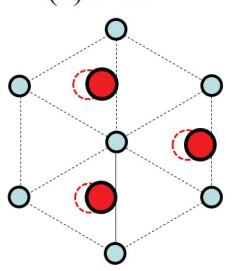

magnetic storage devices [9].

From a theoretical point of view, first-principles calculations have been conducted to investigate the magnetic structure of the fct-like phase for the thin Fe films on the $\mathrm{Cu}(111)$ [10]. However, little is known about the structural and magnetic stability of the Fe bilayers between the bct-like and fct-like phases, and a further understanding of the Fe bilayers based on first-principles calculations is still needed. Here, we investigated structural and magnetic stability of the Fe bilayers on the $\mathrm{Cu}(111)$ substrate by means of first principles calculations. Our total energy calculations indicated the coexistence of the bct-like and fct-like phases, in which the bi-stability of the ferromagnetic (FM) structure and the intra-layer antiferromagnetic (AFM) structures may play an important role.

\section{METHOD AND MODEL}

Calculations were carried out by full-potential linearized augmented plane wave (FLAPW) method [1113] within the generalized gradient approximation (GGA) [14]. LAPW functions with a cutoff of $|\boldsymbol{k}+\boldsymbol{G}| \leq$ 3.9 a.u. $^{-1}$ and muffin-tin (MT) sphere radius of 2.20 a.u. for $\mathrm{Fe}$ and $\mathrm{Cu}$ atoms are used, and lattice harmonics with angular momenta up to $l=8$ are employed to expand the charge and spin densities, potential, and eigenvectors.

In the model of the fct-like phase, the Fe atoms of the surface layer locate on the hollow sites of the subsurface Fe layer, and in the model of the bct-like phase, the Fe atoms of surface are on the bridge sites, as shown in Fig. 1. The $\mathrm{Cu}$ substrates are assumed to consist of three atomiclayers. We have confirmed that the results do not alter by increasing the substrate to five atomic-layers for the FM and AFM structures and three atomic-layers of $\mathrm{Cu}$ may be sufficient to discuss the structural and magnetic stability of the Fe bilayers. Calculations have been performed by changing the position of the surface Fe atoms, with respect to the subsurface layer, along a path from the hollow site $(\delta=0)$ to bridge site $(\delta=1)$, which correspond to the fct-like and bct-like phase, respectively. With an inplane lattice constant that matches to the bulk $\mathrm{Cu}$, all atomic positions along surface normal (z-axis) are fully optimized by using the atomic force calculations.

\footnotetext{
*This paper was presented at the 12th International Conference on Atomically Controlled Surfaces, Interfaces and Nanostructures (ACSIN-12) in conjunction with the 21st International Colloquium on Scanning Probe Microscopy (ICSPM21), Tsukuba International Congress Center, Tsukuba, Japan, November 4-8, 2013.

†Corresponding author: 412m616@m.mie-u.ac.jp
} 
TABLE I: Calculated total energy differences, $\Delta E$, for the fct-like and bct-like phases with magnetic moment alignments of the $\mathrm{FM}$, inter-layer $\mathrm{AFM}$, intra-layer $\operatorname{AFM}(1 \times 2) \mathrm{R} 60^{\circ}$, and intra-layer $\operatorname{AFM}(1 \times 4) \mathrm{R} 60^{\circ}$ structures. The zero energy sets to the bct-like phase with the FM structure.

\begin{tabular}{ccc}
\hline \hline Configuration & $\Delta E$ for fct-like phase $(\mathrm{meV} / \mathrm{Fe}-\mathrm{atom})$ & $\Delta E$ for bct-like phase $(\mathrm{meV} / \mathrm{Fe}-\mathrm{atom})$ \\
\hline $\mathrm{FM}$ & 38 & 0 \\
Inter-layer AFM & 106 & 159 \\
Intra-layer AFM $(1 \times 2) \mathrm{R} 60^{\circ}$ & 100 & 154 \\
Intra-layer AFM $(1 \times 4) \mathrm{R} 60^{\circ}$ & 34 & 60 \\
\hline
\end{tabular}

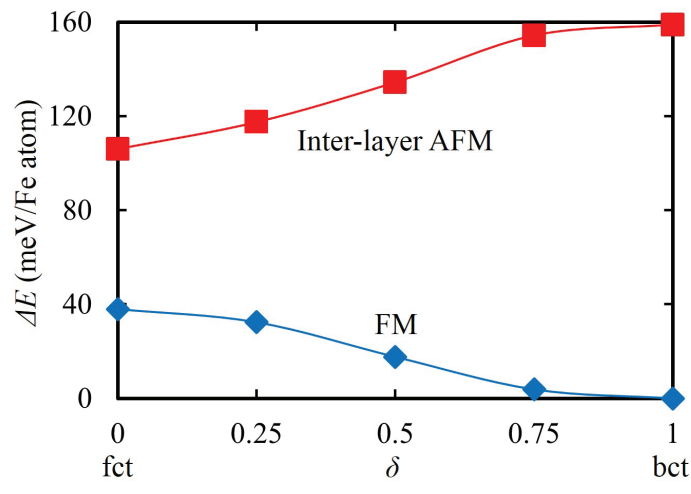

FIG. 2: Calculated total energy differences, $\Delta E$, for Fe bilayers on $\mathrm{Cu}(111)$ substrate. Horizontal axis indicates the lateral positions of the surface Fe atoms, defined in Fig. 1, where $\delta=0$ and 1 correspond fct-like and bct-like phases, respectively.

\section{RESULTS AND DISCUSSION}

Calculated total energy differences, $\Delta E$, as a function of the displacement, $\delta$, for the Fe bilayers on the $\mathrm{Cu}(111)$ substrate are shown Fig. 2. When the FM structure is assumed, an energy minimum appears at $\delta=1$, indicating that the bct-like phase is energetically favorable. However, at $\delta=0$ (the fct-like phase), the total energy curve shows an energy maximum. Thus, there is no (meta-) stable states at $\delta=0$, which is in contrast to the experimental observations of the coexistence of the bct-like and fct-like phases.

In order to examine the instability of the fct-like phase, the density of states (DOS) of the surface Fe atoms and the DOS that projects to the $d_{x y}$ and $d_{x^{2}-y^{2}}$ orbitals for the bct-like and fct-like phases are shown in Fig. 3. The DOS around $E_{\mathrm{F}}$ mainly arises from the minority-spin states, and the majority-spin states are almost fully occupied. For the fct-like phase, the $d_{x y}$ and $d_{x^{2}-y^{2}}$ orbitals degenerate since the surface Fe site has a three-hold symmetry, $C_{3}$. When the Fe atoms displace from the hollow sites, i.e., the $\delta$ deviates from zero, the $C_{3}$ site symmetry degrades. As seen in Fig. 3, the degenerated DOS peak of $d_{x y}$ and $d_{x^{2}-y^{2}}$ orbitals at $-0.1 \mathrm{eV}$ in the fct-like phase $(\delta=0)$ splits when the $\delta$ deviates from zero, and in the bct-like phase $(\delta=1)$, the peak of $d_{x y}$ shifts to $0.4 \mathrm{eV}$ below the $E_{\mathrm{F}}$ so as to reduce the total energy. Thus, the instability of the fct-like phase arises from the Jahn-Teller effects [15].

With regard to the inter-layer AFM structure, where the magnetic moments of the surface and subsurface Fe
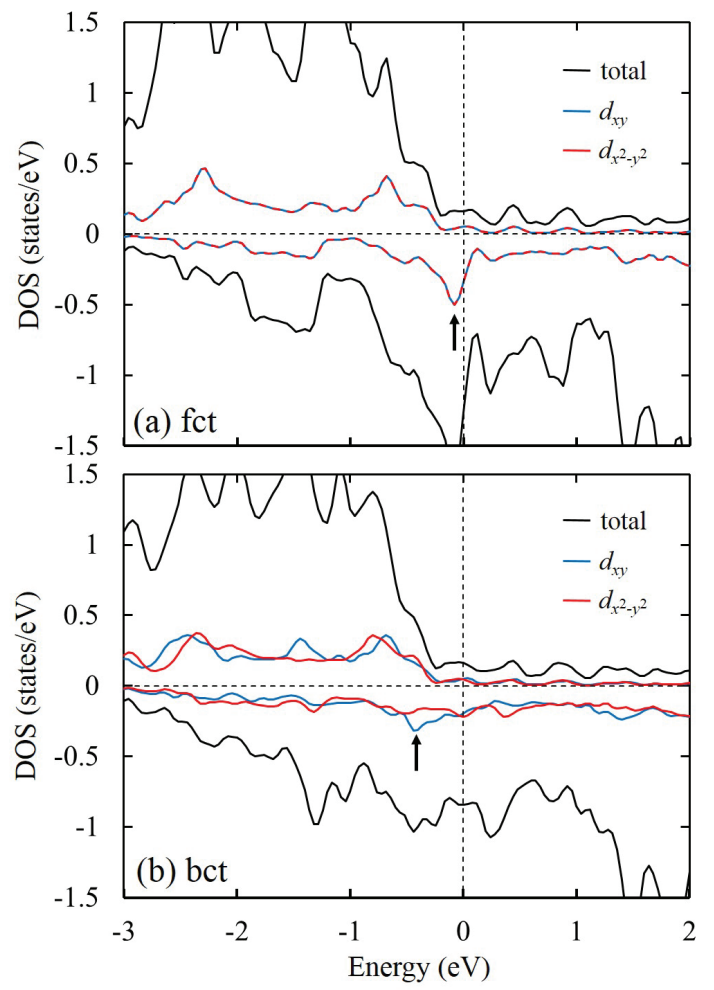

FIG. 3: Calculated density of states (black solid lines) of the surface Fe atom and those projected to the $d_{x y}$ (blue) and $d_{x^{2}-y^{2}}$ orbitals (red) for (a) fct-like and (b) bct-like phases.

layers align in antiparallel each other, we find that the fct-like phase is energetically more stable than the bctlike phase, as shown in Fig. 2. However, the total energy of the fct-like phase in the AFM structure is significantly higher than that of the bct-like phase with the FM structure, by $106 \mathrm{meV} / \mathrm{Fe}$-atom; it may be energetically difficult for both bct-like FM and fct-like AFM phases to coexist, which is still in contrast to the experiments.

Effects of atomic intermixing between the Fe bilayers and the $\mathrm{Cu}$ interface were further considered by extending our calculations where the Fe atoms on the subsurface Fe layer are replaced by $\mathrm{Cu}$ atoms so as to form an ordered $\mathrm{Fe}_{3} \mathrm{Cu}$ structure in the subsurface layer. However, the results indicate that the fct-like phase with the inter-layer AFM structure still be unfavorable compared to the bctlike FM phase, by $104 \mathrm{meV} / \mathrm{Fe}$-atom.

Next, we consider various AFM moment alignments including the intra-layer $\operatorname{AFM}(1 \times 2) \mathrm{R}_{60}^{\circ}$ and $\operatorname{AFM}(1 \times 4)$ $\mathrm{R} 60^{\circ}$ as given in Table I, which correspond the moment alignments of the Fe layers to be antiparallel along the 


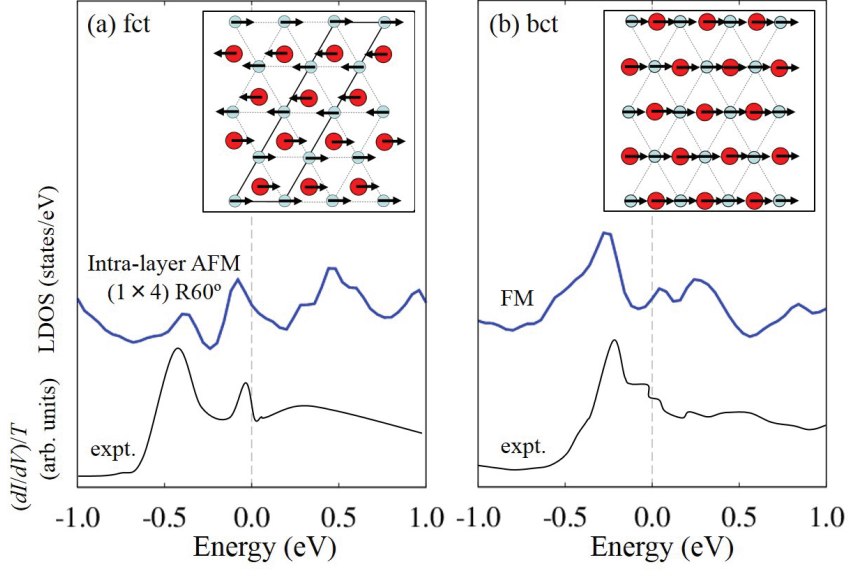

FIG. 4: Calculated local density of states around $E_{\mathrm{F}}$, which were integrated the wave function over a vacuum region for (a) fct-like phase with $\operatorname{AFM}(1 \times 4) \mathrm{R} 60^{\circ}$ and (b) bct-like phase with FM structure. The experimental $d I / d V$ curves obtained by the STS measurements [2] are given by black solid line. Insets in (a) and (b) illustrate their surface structures, where large and small circles indicate $\mathrm{Fe}$ atoms in the surface and subsurface layers and arrows indicate magnetic moment directions.

[01] $]$ direction with a period of single and double atomicrow, respectively. In the fct-like phase, the intra-layer $\operatorname{AFM}(1 \times 4) \mathrm{R}_{60}{ }^{\circ}$ structure is most stable, and furthermore the total energy is lower than that of the bct-like phase when the intra-layer $\operatorname{AFM}(1 \times 4) \mathrm{R} 60^{\circ}$ structure is assumed.

Finally, we compare our results to experiments. Fig- ure 4 shows calculated local density of states (LDOS) around $E_{\mathrm{F}}$, which were obtained by integrating the wave function over vacuum region for the fct-like phase with the intra-layer $\operatorname{AFM}(1 \times 4) \mathrm{R} 60^{\circ}$ structure and the bct-like phases with the FM structure. The experimental $d I / d V$ curves obtained by the scanning-tunneling spectroscopy (STS) measurements [2] are also given in the figure. In the fct-like phase, the present LDOS peaks for the intralayer $\operatorname{AFM}(1 \times 4) \mathrm{R}_{60}{ }^{\circ}$ structure qualitatively cover the STS peaks in experiments, and for the FM structure, a good agreement to experiments was obtained in the bctlike phase.

\section{CONCLUSION}

The structural and magnetic stability of the Fe bilayers was investigated by means of the FLAPW method. The total energy calculations suggest that the bct-like phase is energetically stable for the FM structure but that the fctlike phase is favorable for the intra-layer $\operatorname{AFM}(1 \times 4) \mathrm{R} 60^{\circ}$ structure. The bi-stability of the magnetic structures may play an important role to the coexisting fct-like and bctlike phases as observed in experiments.

\section{Acknowledgments}

Work at Mie University was supported by the Grant-inAid for Scientific Research (No. 20540334) from the Japan Society for the Promotion of Science, and computations were partially performed at ISSP, University of Tokyo.
[1] V. L. Moruzzi, P. M. Marcus, K. Schwarz, and P. Mohn, Phys. Rev. B 34, 1784 (1986).

[2] W. Keune, R. Halbauer, U. Gonser, J. Lauer, and D. L. Williamson, J. Appl. Phys. 48, 2976 (1977).

[3] D. Pescia, M. Stampanoni, G. L. Bona, A. Vaterlaus, R. F. Willis, and F. Meier, Phys. Rev. Lett. 58, 2126 (1987).

[4] U. Gradmann and P. Tillmanns, Phys. Status Solidi A 44, 539 (1977).

[5] M. T. Kief and W. F. Egelhoff, Jr., Phys. Rev. B 47, 10785 (1993).

[6] A. Biedermann, W. Rupp, M. Schmid, and P. Varga, Phys. Rev. B 73, 165418 (2006).

[7] L. Gerhard, T. K. Yamada, T. Balashov, A. F. Takács, R. J. H. Wesselink, M. Däne, M. Fechner, S. Ostanin, A. Ernst, I. Mertig, and W. Wulfhekel, Nat. Nanotechnol. 5, $792(2010)$.

[8] L. Gerhard, T. K. Yamada, T. Balashov, A. F. Takács, R. J. H. Wesselink, M. Däne, M. Fechner, S. Ostanin, A.
Ernst, I. Mertig, and W. Wulfhekel, IEEE Trans. Magn. 47, 1619 (2011).

[9] T. K. Yamada, L. Gerhard, T. Balashov, A. F. Takács, R. J. H. Wesselink, and W. Wulfhekel, Jpn. J. Appl. Phys. 50, 08LA03 (2011).

[10] D. Spišák and J. Hafner, Phys. Rev. B 67, 134434 (2003).

[11] K. Nakamura, T. Ito, A. J. Freeman, L. Zhong, and J. Fernandez-de-Castro, Phys. Rev. B 67, 014405 (2003).

[12] E. Wimmer, H. Krakauer, M. Weinert, and A. J. Freeman, Phys. Rev. B 24, 864 (1981).

[13] M. Weinert, E. Wimmer, and A. J. Freeman, Phys. Rev. B 26, 4571 (1982).

[14] J. P. Perdew, K. Burke, and M. Ernzerhof, Phys. Rev. Lett. 77, 3865 (1996).

[15] H. A. Jahn and E. Teller, Proc. R. Soc. Lond. A 161, 220 (1937). 